\title{
Excited States of Methano-Bridged [10]-, [14]-, and [18]Annulenes. Evidence for Strong Transannular Interaction, and Relation to Homoaromaticity
}

\author{
Harry J. Dewey, ${ }^{1 \mathrm{a}}$ Hans Deger, ${ }^{\mathrm{b}}$ Wolfgang Frölich, ${ }^{\mathrm{b}}$ Bernhard Dick, ${ }^{1 \mathrm{~b}}$ \\ Kenneth A. Klingensmith, ${ }^{1 \mathrm{a}}$ Georg Hohlneicher, ${ }^{* 1 \mathrm{~b}}$ Emanuel Vogel, ${ }^{1 \mathrm{~b}}$ and Josef Michl ${ }^{* 1 \mathrm{a}}$ \\ Contribution from the Department of Chemistry, University of Utah, Salt Lake City, \\ Utah 84112, and the Institute for Organic Chemistry, University of Cologne, Cologne, \\ Germany. Received May 6, 1980
}

\begin{abstract}
Measurements of the absorption, polarized fluorescence, and magnetic circular dichroism of 1,6-methano[10]annulene, 1,6:8,13-bismethano[14]annulene, and 1,6:8,17:10,15-trismethano[18]annulene along with semiempirical $\pi$-electron and all-valence-electron calculations on these and related annulenes are reported. It is concluded that (i) transannular interaction in the methano-bridged annulenes is strong, so that these homoaromatic molecules can be viewed as examples of "arrested transition states" along electrocyclic reaction paths; (ii) in addition to the anticipated $\mathrm{L}_{b}, \mathrm{~L}_{\mathrm{a}}, \mathrm{B}_{\mathrm{b}}$, and $\mathrm{B}_{\mathrm{a}}$ transitions originating in the conjugated $(4 N+2)$-electron perimeter, at least one further low-energy transition appears to be present but its nature is not well understood; (iii) the magnetic circular dichroism of the bridged annulenes is in line with the expectation based on the general theory for cyclic $(4 N+2)$-electron systems and the absolute signs agree with the relative size of orbital energy differences obtained from $\mathrm{CNDO} / \mathrm{S}$ calculations.
\end{abstract}

\section{Introduction}

The stable 1,6-methano[10] annulene (1) has been known for some time. ${ }^{2}$ Recent syntheses of 1,6:8,13-bismethano[14]annulene ${ }^{3}(2)$ and 1,6:8,17:10,15-trismethano[18] annulene ${ }^{4}(3)$ offer a unique opportunity to investigate the electronic properties of a homologous series of bridged annulenes. Earlier studies in annulene spectroscopy, ${ }^{5-9}$ as well as previous measurements on a homologous series of tetra-tert-butylbisdehydroannulenes, ${ }^{10}$ suggest very strongly that expectations based on the simple perimeter model ${ }^{11}$ will be fulfilled also for the series $1-3$, and that the four excited states resulting from electron promotions from the two highest occupied MOs to the lowest two free MOs will dominate the low-energy region in the absorption spectra (weak $\mathrm{L}_{b}$ and $\mathrm{L}_{\mathrm{a}}$ and strong $\mathrm{B}_{\mathrm{b}}$ and $\mathrm{B}_{\mathrm{a}}$ transitions). We find that this is indeed so and direct our attention to the following three problems: (i) can any evidence for or against the presence of transannular interaction between the bridgehead carbons be obtained; (ii) are additional transitions present at low energies and what is their origin; (iii) can the finer details of the spectra, such as the absolute signs in magnetic circular dichroism, be accounted for.

There are good indications $\mathrm{s}^{5,9,12}$ that the bridged annulenes have one or more low-lying excited states in addition to the above four

(1) (a) University of Utah; (b) University of Cologne.

(2) (a) E. Vogel and H. D. Roth, Angew. Chem., Int. Ed. Engl., 3, 228-9 (1964); (b) E. Vogel, Chem. Soc. Spec. Publ., No. 21, 113-47 (1967).

(3) E. Vogel, J. Sombroek, and W. Wagemann, Angew. Chem., Int. Ed. Engl., 14, 564-5 (1975).

(4) (a) W. Wagemann, M. Iyoda, H.-M. Deger, J. Sombroek, and E. Vogel, Angew. Chem., Int. Ed. Engl., 17, 956-7(1978); (b) E. Vogel, H.-M.

Deger, J. Sombroek, J. Palm, A. Wagner, and J. Lex, Ibid., 19, 41-3 (1980).

(5) H.-R. Blattmann, V. Boekelheide, E. Heilbronner, and J.-P. Weber, Helv. Chim. Acta, 50, 68-84 (1967).

(6) H.-R. Blattmann, W. A. Böll, E. Heilbronner, G. Hohlneicher, E. Vogel, and J.-P. Weber, Helv. Chim. Acta, 49, 2017-38 (1966).

(7) W. Grimme, E. Heilbronner, G. Hohlneicher, E. Vogel, and J.-P. Weber, Helv. Chim. Acta, 51, 225-40 (1968).

(8) H.-R. Blattmann, E. Heilbronner, and G. Wagnière, J. Am. Chem. Soc., 90, 4786-9 (1968); U. P. Wild in "Excited States in Organic Chemistry and Biochemistry", B. Pullman and N. Goldblum, Eds., D. Reidel Publishing Co., Dordrecht, Holland, 1977, pp 387-95. (1976)

(10) J. Tanaka, M. Tanaka, M. Morita, M. Nakagawa, and M. Iyoda, Bull. Chem. Soc. Jpn., 51, 3458-61 (1978).

(1i) J. R. Platt, J. Chem. Phys., 17, 484-95 (1949), W. Moffitt, ibid., 22 320-33, 1820-29 (1954); E. Heilbronner and J. N. Murrell, Mol. Phys., 6 $1-18(1963)$.

(12) J. Spanget-Larsen and R. Gleiter, Helv. Chim. Acta, 61, 2999-3013 (1978). which are readily accounted for as involving the excitation of the $\pi$ electrons of the perimeter. Several possible assignments were considered in previous work, ${ }^{9,12}$ but the situation remained unsatisfactory. Recent experimental results for $5,6,9$, and $10^{13}$ cast serious doubt on one of the proposals ${ }^{9}$ concerning the origin of the lowest energy one among these "extra" transitions (labeled $A$ in ref 9). This proposal was also questioned recently 12 as being incompatible with results of CNDO calculations.

\section{Experimental Section}

Materials. Preparation of pure samples of 1-3 has been described elsewhere. ${ }^{2-4}$ 3-Methylpentane (3-MP, Phillips Petroleum Co.) and 2methyltetrahydrofuran (2-MTHF) were refluxed with sodium, distilled, and passed over an $\mathrm{Al}_{2} \mathrm{O}_{3}-\mathrm{AgNO}_{3}$ column. Other solvents were spectrograde quality.

Spectroscopy. Absorption and emission spectra in rigid glass were taken in 2-mm Suprasil cells immersed in a quartz Dewar with Suprasil windows, filled with filtered liquid nitrogen. Absorption spectra were measured on a Cary 17 spectrophotometer, polarized fluorescence and fluorescence excitation spectra on a home-made instrument assembled from a $1-\mathrm{kW}$ xenon lamp, a double $0.5-\mathrm{m}$ excitation monochromator (Spex 1302), two Glan prisms, an $0.75-\mathrm{m}$ analyzing monochromator (Spex 1702), a cooled S20 response photomultiplier, and a single-photon counting system. Fluorescence lifetimes were measured using a Molectron UV 1000 nitrogen laser pumped dye laser with a 7-8-ns pulsewidth and a Tektronix 7844 oscilloscope with a 7A19 amplifier and 7B80 time base.

Magnetic circular dichroism was measured at room temperature using a JASCO $500 \mathrm{C}$ spectropolarimeter equipped with a $15-\mathrm{kG}$ electromagnet, wavelength calibrated with a holmium oxide film filter, and scale calibrated with the $\mathrm{CD}$ of $d$-camphorsulfonic acid and the MCD of naphthalene.

\section{Calculations}

$\pi$-Electron Calculations. We have performed a variety of PPP calculations on 1-3, 9-14, and the hypothetical [10]- and [14]annulenes with regular polygon geometries, using either all singly excited configurations (SCI) or all singly and selected doubly excited ones (SDCI), and employing various parameter choices. The conclusions from all these calculations are the same and we only reproduce here the results obtained with the parameter set of ref 14: $\alpha_{\mathrm{C}}=11.16 \mathrm{eV}, \beta_{\mathrm{CC}}(\mathrm{R})=[-2.40+3.21(R(\AA)-1.397)]$ $\mathrm{eV}, \gamma_{00}=11.13 \mathrm{eV}$. For the two-center repulsion integrals the Mataga formula ${ }^{15}$ was used for SCI and the Ohno formula ${ }^{16}$ for

(13) H. J. Dewey, V. Boekelheide, and J. Michl, to be published

(14) K. Schulten, I. Ohmine, and M. Karplus, J. Chem. Phys., 64, 4422-41 (1976)

(15) K. Nishimoto and N. Mataga, Z. Phys. Chem., 12, 335-8 (1957); 13 , 140-43 (1957). 


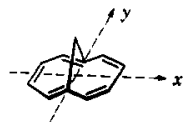

1
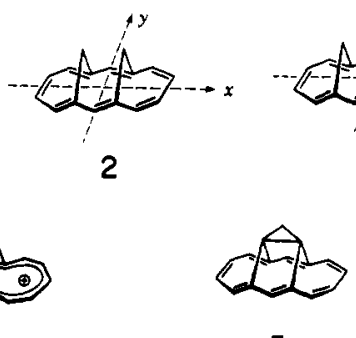

4

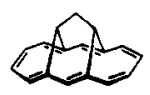

7

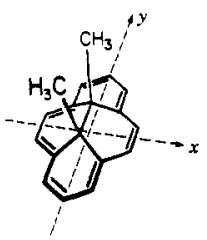

9

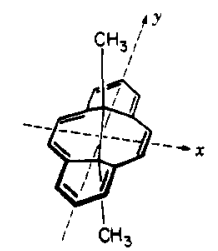

10

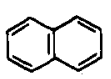

11
12

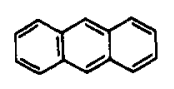

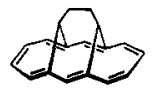

8
3

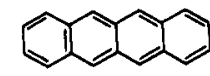

13<smiles>c1ccccccccccccc1</smiles>

14

SDCI. ${ }^{17}$ In SDCI 100 lowest energy configurations were used for the $10-\pi$-electron systems, 150 for the 14- $\pi$-electron systems, and 200 for the 18- $\pi$-electron systems. The geometries of $1^{18}$ and $2^{19}$ were taken from X-ray data; that of 3 was estimated from the former two. In the calculations for 9-14, all bond lengths were assumed to be $1.4 \AA$ and all $\mathrm{C}-\mathrm{C}-\mathrm{C}$ angles $120^{\circ}$. The resonance integrals of twisted bonds were reduced by the factor $\cos \alpha$, where $\alpha$ is the twisting angle. Transannular interaction was introduced into 1-3 by introducing cross-ring resonance integrals between the bridgehead positions; their value was varied from 0 to -2.4 $\mathrm{eV}$.

All-Valence Electron Calculations. Calculations by the CNDOCI method were performed using singly and doubly excited configurations ( 200 configurations for 1, 250 for 2,300 for 3 ). All computational details, especially the selection of the lowest energy configurations, are the same as those in ref 17.

\section{Results}

The low-temperature absorption, fluorescence, and corrected excitation spectra of the methano-bridged annulenes 1-3 are shown in Figures 1-3 along with the plots of the degree of polarization in excitation and fluorescence spectra. Fluorescence lifetimes are given where they could be measured. Room-temperature absorption spectra and magnetic circular dichroism are shown in Figures 4-6.

(16) K Ohno, Theor Chim Acta, 2 219-27 (1964)

(17) B. Dick and G. Hohlneicher, Theor. Chim. Acta, 53, 221-51 (1979)

(18) M. Dobler and J. D. Dunitz, Helv. Chim. Acta, 48, 1429-40 (1965).

(19) R. Destro, T. Pilati, and M. Simonetta, Acta Crystallogr., Sect. B, 33, 940-42 (1977).
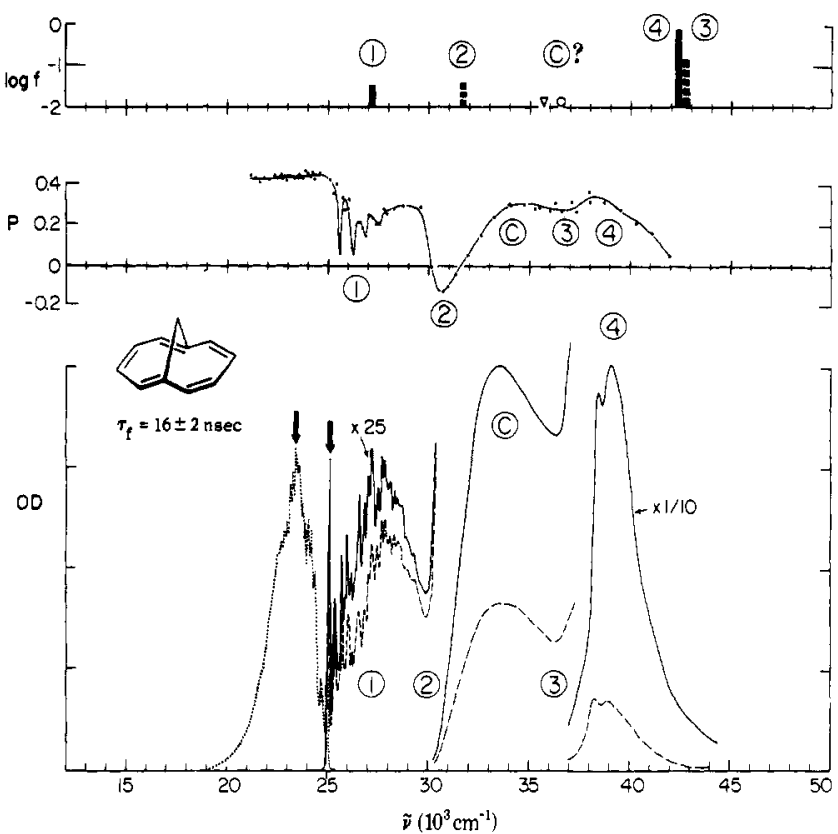

Figure 1. 1,6-Methano[10]annulene (1) in 3-methylpentane glass at 77 K. Bottom: absorption (full line), emission (dotted line, not corrected for instrumental response), and excitation (dashed line, corrected for instrumental response). Center: degree $P$ of polarization of excitation and emission, monitored at positions indicated by the arrows at the bottom. Top: calculated (CNDO-CI) transition energies, oscillator strengths $(f)$ and polarizations ( $x$, full; $y$, dashed bar; $z$, empty bar). Weak transitions are shown as dark dots ( $x$-polarized), half-filled dots $(y$-polarized), empty dots ( $z$-polarized), or triangles (symmetry-forbidden).
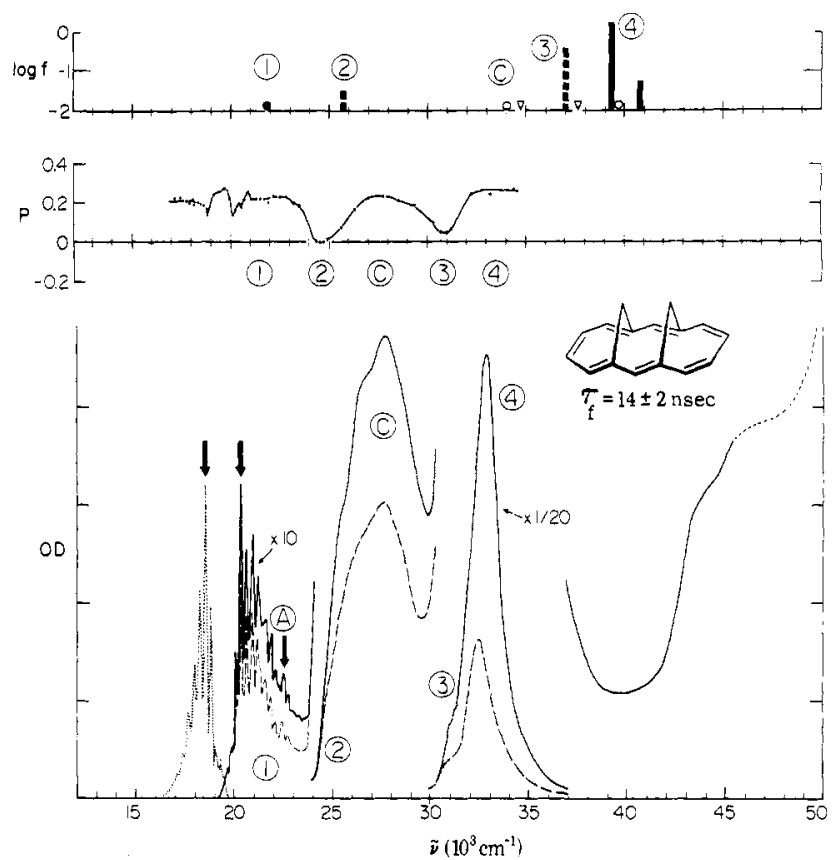

Figure 2. 1,6:8,13-Bismethano[14]annulene (2) in 3-methylpentane glass at $77 \mathrm{~K}$. See caption to Figure 1.

The expected four fundamental electronic transitions are evident in the spectra of all three compounds and are labeled in the figures as $1\left(\mathrm{~L}_{\mathrm{b}}, x\right.$-polarized $), 2\left(\mathrm{~L}_{\mathrm{a}}, y\right.$-polarized $), 3\left(\mathrm{~B}_{\mathrm{a}}, y\right.$-polarized $)$, and $4\left(\mathrm{~B}_{b}, x\right.$-polarized). The assignment is based on transition intensities and relative polarizations as well as on analogy to previously studied annulenes, ${ }^{9}$ which was also used to assign the absolute polarizations. The $\mathrm{L}_{b}$ transition has a long-axis polarized origin but shows mixed polarization beyond the origin, with clear evidence of intensity borrowing, presumably from the nearby $\mathrm{L}_{\mathrm{a}}$ transition. The vibrational structure is well resolved in the po- 

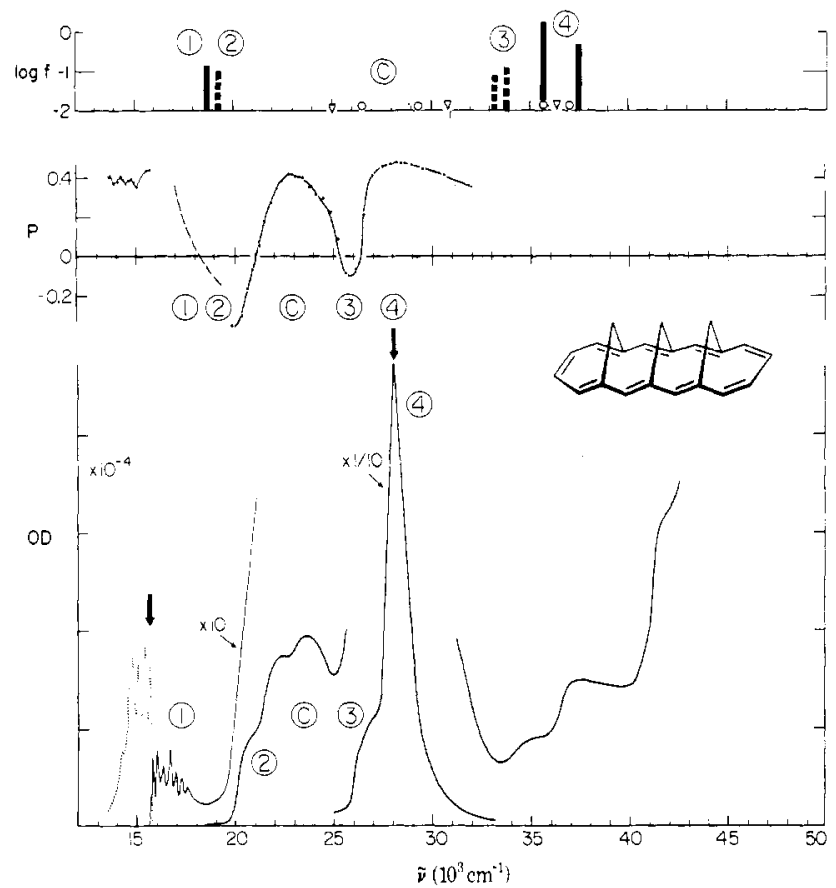

Figure 3. 1,6:8,17:10,15-Trismethano[18]annulene (3) in 2-methyltetrahydrofuran glass at $77 \mathrm{~K}$. See caption to Figure 1 . The dashed part of the polarization curve was measured in ethanol glass.
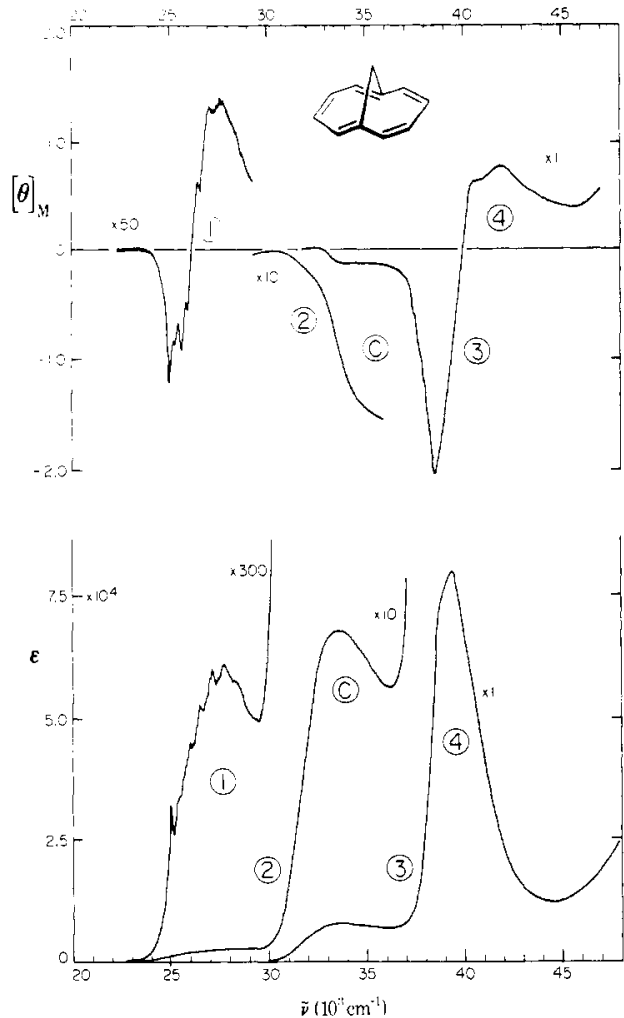

Figure 4. 1,6-Methano[10]annulene (1) in 3-methylpentane, room temperature. Bottom: absorption; top: magnetic circular dichroism. Molar ellipticity per unit field strength in units of $\operatorname{deg} \mathrm{L} \mathrm{m}^{-1} \mathrm{~mol}^{-1} \mathrm{G}^{-1}$.

larization curve of $\mathbf{1}$, positive at the vibrational peaks and dipping toward negative values in the valleys between them. It is only poorly resolved in the polarization curve of 2 which shows a uniformly low value, presumably due to overlap of vibronic peaks of different polarizations. The fluorescence of 3 is weak, its solubility is poor, and no reliable polarization measurements were possible in the region of the weak first band in 2-methyltetrahydrofuran solvent. Higher concentrations could be used in ethanol and the observed polarization curve again shows the presence of vibronic mixing. In view of the complexity of the

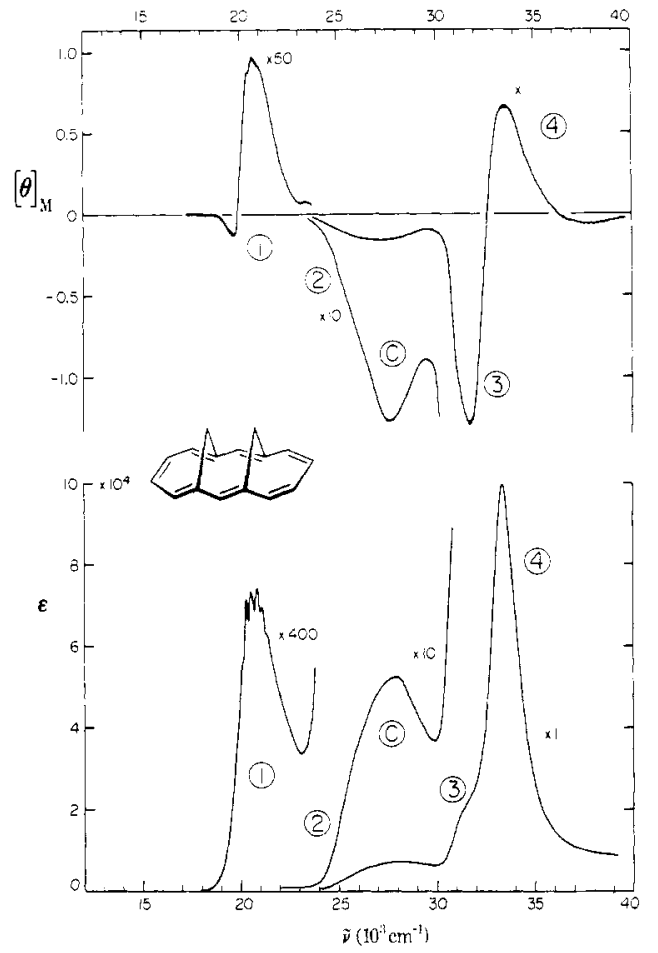

Figure 5. 1,6:8,13-Bismethano[14]annulene 2 in 3-methylpentane, room temperature. See caption to Figure 4.
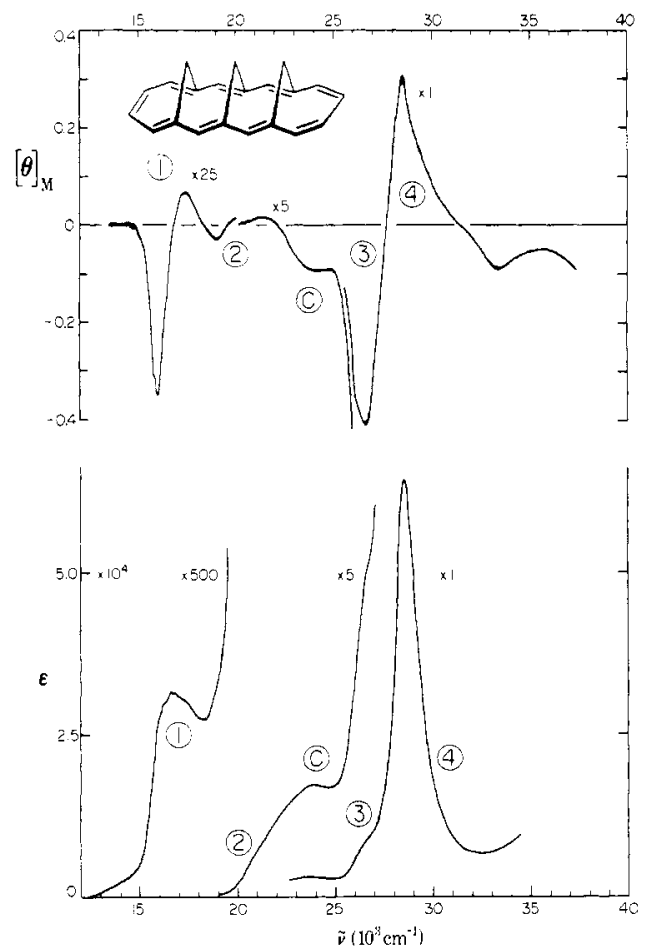

Figure 6. 1,6:8,17:10,15-Trismethano[18]annulene 3 in tetrahydrofuran, room temperature. See caption to Figure 4.

spectra and the poor resolution, no attempt at an analysis of the vibrational structure was made.

The corrected excitation spectra follow the absorption spectra at low energies, but the efficiency of the fluorescence falls off at excitation energies above that of the $L_{a}$ transition. The fluorescence spectra are fair mirror images of the first absorption band, particularly for 2 and 3 . The $0-0$ component in absorption and emission is strong in all cases except 2 , where it is curiously weak, giving the band a distinct Franck-Condon-forbidden appearance. In this regard, $\mathbf{2}$ is similar to 8 and different from $5-7.9$

The MCD spectra clearly show the presence of transitions $1-4$, and in the case of 1,6-methano[10] annulene, whose MCD curve 

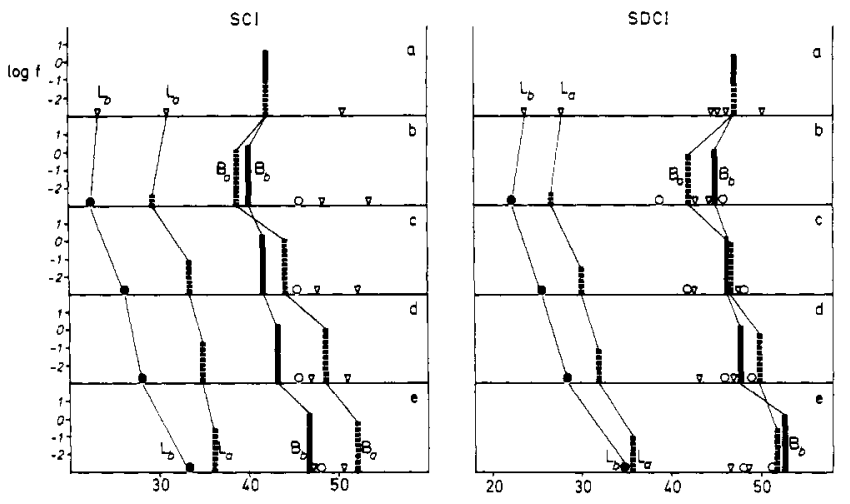

$$
\tilde{\nu}\left(10^{3} \mathrm{~cm}{ }^{-1}\right)
$$

Figure 7. 1,6-Methano[10]annulene 1: $\pi$-electron calculation of transannular interaction effects. Transition energies, intensities, and polarizations are shown using the symbols explained in the caption to Figure 1. (a) $D_{10 h}[10]$ annulene; (b) [10]annulene with the experimental geometry of the perimeter of $\mathbf{1}$, no transannular interaction; (c) as in b, but the resonance integral for transannular 1,6 interaction is set at $\beta_{\text {trans }}=$ $-0.96 \mathrm{eV}$; (d) as in b, but $\beta_{\text {trans }}=-1.68 \mathrm{eV}$; (e) naphthalene 11. In the planar polyacenes, empty circles (triangles) mark transitions to $A_{g}\left(B_{g}\right)$ states.

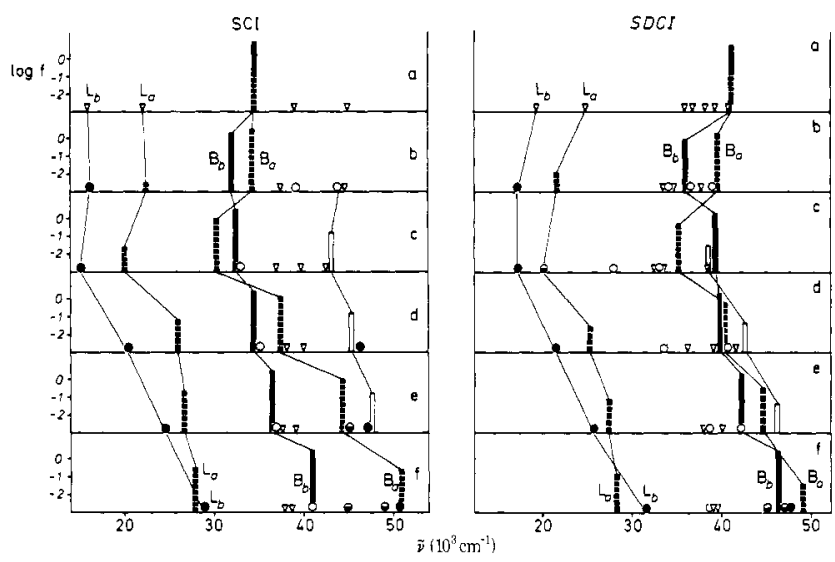

Figure 8. 1,6:8,13-Bismethano[14] annulene (2); see caption to Figure 7. (a) $D_{14 h}$ [14] annulene; (b) [14] annulene with the idealized dihydropyrene geometry of 9 and 10; (c) [14]annulene with the experimental geometry of the perimeter of 2, no transannular interaction; (d) as in c, but $\beta_{\text {trans }}=-0.96 \mathrm{eV}$; (e) as in $\mathrm{c}$, but $\beta_{\text {trans }}=-1.68 \mathrm{eV}$; (f) anthracene 12.

was already reported earlier, ${ }^{20}$ they actually provide the most convincing evidence for the existence of transition 3 , which is not obvious in absorption and is only hinted at in the polarization curve. The MCD of transition 1 is bisignate in each case, and this distinguishes the [14]annulene 2 from the previously studied ${ }^{9}$ related [14]annulenes 5-8.

In addition to the four obviously present transitions $1-4$, up to two other transitions ( $\mathrm{A}, \mathrm{C}$ ) are assigned tentatively in the figures in analogy to the A, B, C transitions which appear to be present in the bridged [14] annulenes 5-8. ${ }^{9}$ Of the two long-axis $(x)$ polarized transitions, $C$ is strong and clearly present in all of the annulenes, $1-3$ as well as 5-8 and even $9^{13}$ and $10,{ }^{12}$ but the presence of $\mathrm{A}$ is obvious only in some cases, as discussed in more detail below.

Figures 7-9 show the results of our $\pi$-electron calculations for the [10]-, [14]-, and [18] annulene perimeters. In each case, the top row shows the predictions for an idealized perimeter with no transannular interactions, and the bottom row shows those for the polyacene $(11-13)$ which is obtained from the perimeter by introducing one, two, or three cross-links, respectively. The middle rows display the calculated effects of a gradual introduction of the cross-links, starting with a perimeter distorted into the non-

(20) B. Briat, D. A. Schooley, R. Records, E. Bunnenberg, C. Djerassi, and E. Vogel, J. Am. Chem. Soc., 68, 4691-7 (1968).
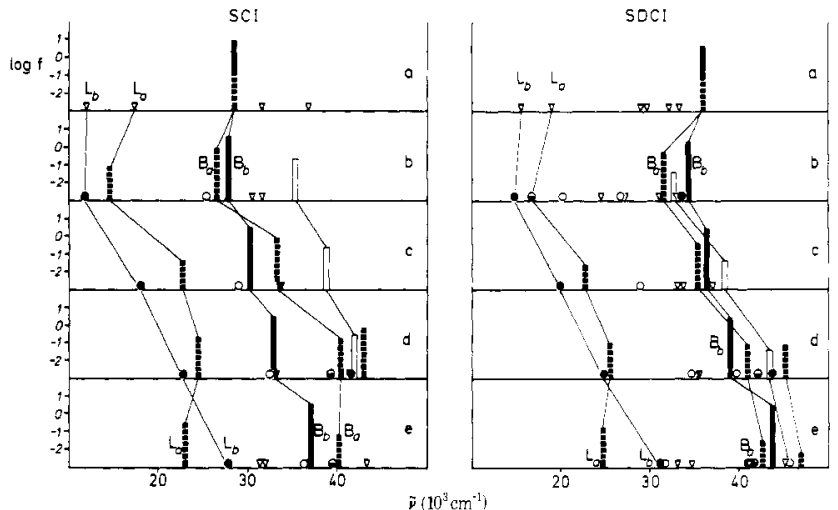

Figure 9. 1,6:8,17:10,15-Trismethano[18]annulene (3); see caption to Figure 7. (a) $D_{6 h}[18]$ annulene 14 ; (b) [18]annulene with the extrapolated geometry of the perimeter of 3 , no transannular interaction; (c) as in $\mathrm{b}$, but $\beta_{\text {trans }}=-0.96 \mathrm{eV}$; (d) as in $\mathrm{b}$, but $\beta_{\text {trans }}=-1.68 \mathrm{eV}$; (e) tetracene 13.

planar elongated shape characteristic of the methano-bridged annulenes but free of any transannular bonding and proceeding in several steps toward the fully bonded polyacene extreme. The geometry of the ideal perimeter on the top was taken to be that of a regular $D_{n h}$ polygon for the [10]- and [14]annulenes; for these, no experimental data exist. For the [14]annulene perimeter, results for a pyrene-like shape as found in $\mathbf{9}$ and $\mathbf{1 0}$ are also shown. For the [18] annulene, $D_{6 h}$ geometry of formula 14 was assumed. In this instance, experimental data are available ${ }^{8}$ and permit a direct verification of the trend predicted for all three cases, both at the SCI and the SDCI levels: introduction of transannular interactions should increase all four excitation energies, particularly those of the $\mathrm{L}_{b}$ and $\mathrm{B}_{\mathrm{a}}$ transitions. An analysis of this trend in terms of simple perturbation theory for the case of [14]annulenes has been given in ref 9.

The CNDO-SDCI results are compared with the experimental spectra in Figures 1-3. They do not differ significantly from the $\pi$-electron results. Although the calculated and observed energies do not always agree perfectly, both sets of calculations account quite well for transitions $1-4$. The CNDO-SDCI method reproduces correctly the shifts of the B bands within the series 1-3, but overestimates their excitation energy. Neither set of calculations provides assignments for the possibly present bands $A$ and $B$, nor for the clearly present strong absorption assigned as $C$. As noted before, 9,12 we find again that the use of doubly excited configurations in the $\mathrm{CI}$ procedure brings several transitions below the $B_{b}$ and $B_{a}$ bands, but their intensity is exactly zero in models which preserve the pairing theorem, such as our $\pi$-electron calculations, and it is almost exactly zero in the CNDO calculation as well, although this model does away with pairing symmetry (actually, usually too much so ${ }^{21}$ ).

\section{Discussion}

Transannular Interaction. The existence of transannular interactions in methano-bridged annulenes has been previously deduced from an analysis of the electronic spectra of $1,{ }^{6}$ of the bridged cation $4,{ }^{7}$ and of a series of bridged [14] annulenes $5-8,{ }^{9}$ and also from the analysis of the photoelectron spectra of this class of compounds.22 The most likely value of the transannular resonance integral was estimated at about $40 \%$ of that between $2 \mathrm{p}_{z}$ orbitals on neighbors in benzene.

These calculations have not found universal acceptance. For instance, a recent review article on homoaromaticity $y^{23}$ questions whether any transannular interaction is at play in 1 or related bridged annulenes. The existence of such doubts is curious and

(21) J. H. Obbink and A. M. F. Hezemans, Chem. Phys, Lett., 50, 133-7 (1977); cf. M. J. Robey, I. G. Ross, R. V. Southwood-Jones, and S. J. Strickler, Chem. Phys., 23, 207-16 (1977).

(22) (a) R. Boschi, W. Schmidt, and J.-C. Gfeller, Tetrahedron Lett., 4107-10 (1972); (b) C. Batich, E. Heilbronner, and E. Vogel, Helv. Chim. Acta, 57, 2288-2306 (1974).

(23) L. A. Paquette, Angew. Chem., Int. Ed. Engl., 17, 106-17 (1978). 
is perhaps due to excessive reliance on the results of NMR measurements alone in the evaluation of such interactions. The NMR method is undisputably extremely useful in investigations of cyclic conjugation, but like other "all-electron properties", it loses its ability to detect extended conjugation rapidly once the interactions in question are not essential for maintaining cyclic conjugation, particularly if they decrease significantly below the standard "aromatic" value. "One-electron properties" like optical and photoelectron spectra, interpretable in terms of properties of individual molecular orbitals, respond to transannular interaction and similar extended conjugation phenomena already in first rather than in higher order, and in this regard are more suitable for these investigations.

The results of optical ${ }^{6,7,9}$ and photoelectron ${ }^{22}$ spectroscopy of methano-bridged annulenes have not been questioned nor have alternative interpretations of the data been proposed. A possible reason for the apparent lack of confidence in the conclusions drawn is the fact that models for ideal planar [10]- and [14]annulenes free of transannular interaction or possible hyperconjugation with saturated bridges are not available, so that the interpretations of optical and photoelectron data had to rely on comparison with calculations to a degree which might appear to be excessive.

From this point of view, the present results provide a unique opportunity to compare the spectral properties of an ideal annulene, namely, the [18] annulene $14,{ }^{8}$ with those of a trismethano-bridged [18] annulene 3 , and with a known compound in which the three transannular interactions have become full-fledged aromatic bonds, namely, tetracene $13 .^{24}$ On going from 14 to 13 , the $L_{b}$ transition energy increases from the $13000 \mathrm{~cm}^{-1}$ observed in 14 to at least $21000 \mathrm{~cm}^{-1}$ in 13 , where it is buried under the $L_{a}$ transition, which in turn did not change its energy much $\left(21900 \mathrm{~cm}^{-1}\right.$ in 14,20800 $\mathrm{cm}^{-1}$ in 13). The enormous sensitivity of the energy of the $L_{b}$ transition to transannular interaction and the eventual change in state order are exactly as predicted by theory (Figure 9). In the methano-bridged [18] annulene 3 , the energy of the $\mathrm{L}_{b}$ transition is $15700 \mathrm{~cm}^{-1}$ and that of the $\mathrm{L}_{\mathrm{a}}$ transition is about $20000 \mathrm{~cm}^{-1}$. The considerable blue shift of the $\mathrm{L}_{b}$ transition in 3 relative to $13\left(2700 \mathrm{~cm}^{-1}\right)$ thus provides clear evidence for transannular interaction in 3. The behavior is of exactly the same type as found previously, 6,7,9,22 for methano-bridged [10]- and [14] annulenes, estimating the properties of the unperturbed perimeter from calculations.

It is difficult to put the argument in quantitative terms without relying on calculations to some degree, for at least three reasons: (i) the exact energy of the $L_{b}$ transition in $\mathbf{1 3}$ is unknown; (ii) the relation between the $L_{b}$ transition energy and the "per cent bond" which exists across a bridged position need not be linear (calculations suggest that it is nearly linear, cf. Figures 7-9); (iii) while the neglect of the possible inductive and hyperconjugative effects of the methano bridges on the $\mathrm{L}_{b}$ shift is reasonable (e.g., tetramethyl substitution shifts the $\mathrm{L}_{b}$ band of 10 by only ca. $300 \mathrm{~cm}^{-1}$ and to the red ${ }^{12}$ ), the twisting of perimeter bonds which is known to be present in all methano-bridged annulenes ${ }^{18,19,25,26}$ cannot be ignored since it must lower the $\mathrm{L}_{b}$ transition energy and can well reduce the observed value of the blue shift substantially. Actually, the fact that a blue shift is observed in spite of the twisting is an excellent indication that the transannular interaction is quite strong. The origin of the undoubtedly present red-shifting effect of the twisting is the same as in the case of twisted olefins or small ring [n]paracyclophanes; ${ }^{27}$ carried to its extreme, the twisting

(24) R. Kiessling, G. Hohlneicher, and F. Dörr, Z. Naturforsch, A, 22 1097-1108 (1967).

(25) N. A. Bailey and R. Mason, Chem. Commun., 1039-42 (1967); T. Pilati and M. Simonetta, Acta Crystallogr., Sect. B, 32, 1912-13 (1976); P. Ganis and J. D. Dunitz, Helv. Chim. Acta, 50, 2369-78 (1967); A. Gavezzotti, A. Mugnoli, M. Raimondi, and M. Simonetta, J. Chem. Soc,, Perkin Trans. 2, 425-31 (1972); C. M. Gramaccioli, M. Mugnoli, T. Pilati, M. Raimondi, and M. Simonetta, Acta Crystallogr., Sect. B, 28, 2365-70 (1972); R. Bianchi, A. Mugnoli, and M. Simonetta, ibid., 31, 1283-7 (1975); R. Bianchi, G. Casalone, and M. Simonetta, ibid., 31, 1207-09 (1975); A. Mugnoli and M. Simonetta, ibid., 30, 2896-8 (1974).

(26) R. Bianchi, G. Morosi, A. Mugnoli, and M. Simonetta, Acta Crystallogr., Sect. B, 29, 1196-1208 (1973). breaks the unsaturated systems into subunits in such a way that a biradical results (see ref 9 for additional detail).

Our calculations for 1-3 indicate that in each case the transannular resonance integrals must be about $40 \%$ of the standard value for aromatic carbons in order to overcome the effects of the twisting and produce the observed blue shift (Figures 7-9). This agrees with previous estimates in other methano-bridged annulenes and with the magnitude of the overlap between the orbitals involved.

It seems to us that in the case of well-defined geometry, such as the methano-bridged annulenes, many of which have been subjected to $X$-ray structure analysis, ${ }^{18,19,25,26}$ the question is not "can one prove that there is any transannular interaction?" but rather, it is purely rhetorical: "how could there possibly fail to be transannular interaction between two valence AOs located near each other and directed at each other so that their overlap must be significant no matter which exact form is chosen to represent them?"

At times, an objection is raised to the use of properties like optical and photoelectron spectra for purposes such as analysis of conjugation effects or search for transannular interaction, since these properties depend not only on the ground state but also on an excited or ionized state of the molecule. However, this is irrelevant; both in optical and in photoelectron spectra, it is possible to observe vertical transitions, i.e., transitions which do not involve a change in molecular geometry. Although valence-shell excitations and ionizations will generally change the optimum AO basis set somewhat, two valence AOs which overlapped significantly in the ground state will still do so in the excited or ionized state and vice versa, as long as the molecular geometry is the same. Thus, there must be very little difference in the degree of transannular interaction between AOs in the individual vertically excited or ionized states of the molecule, but there, of course, can be much difference in the degree to which the presence of such interaction is reflected in the energy and other properties of the individual electronic states.

Homoaromaticity. In view of the significant transannular interactions in the methano-bridged annulenes $1-3$, it would be quite proper to refer to them as homonaphthalene, bishomoanthracene, and trishomotetracene, respectively, by analogy to other homoaromatic species. ${ }^{23}$ Molecules of this kind are probably the best documented examples of "arrested transition states", i.e., transition states stabilized by suitable structural factors to such a degree that a negative activation energy is required to reach them from either end of a normal reaction path, e.g., in the case of 1 , an electrocyclic path. ${ }^{28}$ Structural variations are capable of shifting the location of the energy minimum along the reaction path. For instance, the equilibrium geometry of the 11,11-dimethyl derivative of 1 is almost that of a cyclopropane, with a $1.8-\AA$ separation between the two bridgehead carbons, ${ }^{26}$ while in most cases this distance is of the order of $2.25 \AA$.

The annulenes 2 and 3 are particularly intriguing since they can be thought of as transition states with two and three partially made bonds, respectively, corresponding to a juncture of several electrocyclic and Cope rearrangement paths. In this connection, one wonders whether the recent discussions of the elusiveness of neutral homoaromaticity 29,30 are not excessively pessimistic. Perhaps, in addition to the donor-acceptor interaction possibility discussed in ref 30 , a chance may yet exist that suitable steric and substituent constraints will be devised to force an "arrested transition state", and neutral homoaromaticity, even on a $2+2$ +2 cycloaddition path. In our opinion, the methano-bridged annulenes, although clearly possessing stabilizing homoaromatic

(27) N. L. Allinger, J. T. Sprague, and T. Liljefors, J. Am. Chem. Soc., 96, $5100-5104$ (1974), and references cited therein. (1975).

(29) G. C. Christoph, J. L. Muthard, L. A. Paquette, M. C. Böhm, and R. Gleiter, J. Am. Chem. Soc., 100, 7782-4 (1978); L. A. Paquette, R. A Snow, J. L. Muthard, and T. Cynkowski, ibid., 101, 6991-6 (1979).

(30) K. N. Houk, R, W. Gandour, R. W. Strozier, N. G. Rondan, and L. A. Paquette, J. Am. Chem. Soc., 101, 6797-802 (1979), 
interactions (the requisite transannular bond orders in the parent annulenes are all positive), are primarily examples of neutral aromaticity rather than neutral homoaromaticity, since they possess an uninterrupted conjugated perimeter and would remain conjugated and aromatic even if all the "homo" interactions were removed.

Additional Transitions. In addition to the clearly present and readily interpreted transitions $1\left(\mathrm{~L}_{b}\right), 2\left(\mathrm{~L}_{a}\right), 3\left(\mathrm{~B}_{\mathrm{a}}\right)$, and $4\left(\mathrm{~B}_{\mathrm{b}}\right)$, other low-energy transitions are present in the spectra of the bridged annulenes. In ref 9 , up to three of them were identified tentatively and labeled A, B, and C. Not all of these are clearly present in each of the annulenes. The long-axis $(x)$ polarized transition $\mathrm{A}$ is prominent in the spectra of $5,{ }^{9} 6,{ }^{9} 9,{ }^{13}$ and $10^{5,12}$ but not in those of $7,{ }^{9} 8,{ }^{9}$ nor the presently investigated 1 and 3 . Its presence in the spectrum of $\mathbf{2}$ is questionable and it has been marked with a question mark in Figure 2 . It has been suggested ${ }^{9}$ that the existence of transition $\mathrm{A}$ is due to hyperconjugation with the saturated bridge, and more specifically to an excitation of $\sigma$ $\rightarrow \pi^{*}$ type. This suggestion was not confirmed by recent CNDOCI calculations, ${ }^{12}$ nor is it supported by those reported presently. Very recently obtained high-resolution single-site-selected spectra of matrix-isolated $\mathbf{5}$ and $\mathbf{6}$ suggest that $\mathrm{A}$ is a part of the vibronic structure of transition 1, in spite of appearances to the contrary in more usual types of spectra. ${ }^{13}$ The short-axis $(y)$ polarized transition $B$ is prominent only in the spectrum of 5 . Its possible presence is indicated by no more than a shoulder in the polarization curves of $6,{ }^{9} 7,{ }^{9}$ and $8 .{ }^{9}$ In the case of $5, \mathrm{CNDO}-\mathrm{CI}$ calculations ${ }^{12}$ agree with the tentative assignment ${ }^{9}$ to a $\sigma \rightarrow \pi^{*}$ transition due to hyperconjugation. Since transitions A and $\mathbf{B}$ are not obvious in the spectra of 1-3, we shall not deal with them further.

Transition $C$ is clearly present in the polarized absorption spectra of all of the methano-bridged annulenes, but is curiously absent in their MCD. It could again involve the electrons of the saturated bridge, ${ }^{9}$ but CNDO-CI calculations do not lend support to this notion (ref 12 and present calculations). It could also be due to one of the several transitions which are calculated to lie below the $B_{a}$ and $B_{b}$ bands when doubly excited configurations are used in the $\mathrm{CI}$ procedure ( $\pi$ calculations of ref 9 and Figures $7-9$, all-valence electron calculations of ref 12 and Figures 1-3). However, these transitions have negligible or zero intensity in all of the calculations performed so far, while the observed long-axis polarized absorption is quite strong. A convenient and perhaps correct way out of the dilemma is to assign all of the observed intensity to vibronic borrowing by one or more of these "forbidden" transitions from the $B_{b}$ transition. Given that such massive vibronic intensity stealing needs to be invoked, it is natural to ask whether the presence of any "forbidden" transitions in this region is demanded by the experimental data at all and whether it is not more economical to assign the vibronic activity to the already welldocumented transition 2.

Signs in Magnetic Circular Dichroism. In a previous investigation ${ }^{9}$ of the bridged annulenes 5-8, the observed MCD signs of the $B_{a}$ and $B_{b}$ transitions were taken as an indication that hyperconjugation with the bridge is significant and no attempt was made to interpret the signs of the $\mathrm{L}_{\mathrm{a}}$ and $\mathrm{L}_{b}$ transitions. The qualitative features of MCD spectra of cyclic $\pi$-electron systems derived from $(4 N+2)$-electron $[n]$ annulene perimeters have since become much better understood and the MCD signs of all four $\mathrm{L}$ and $\mathrm{B}$ transitions in 1-3 as well as 5-8 are easily accounted for in terms of the general theory, ${ }^{31}$ as follows. The signs of the two $\mathrm{B}$ transitions are those expected of the $\mu^{-}$contributions resulting from their mutual magnetic mixing, similarly as in naphthalene, anthracene, and their derivatives. ${ }^{32}$ The $\mu^{-}$contributions to the $B$ terms of the $L$ transitions are both expected to be weakly positive, resulting in negative peaks in the MCD

(31) J. Michl, J. Am. Chem. Soc., 100, 6801-24 (1978)

(32) M. Vašäk, M. R. Whipple, and J. Michl, J. Am. Chem Soc, 100 , 6838-43 (1978); M. R. Whipple, M. Vašăk, and J. Michl, ibid., 100, 6844-52 (1978); R. P. Steiner and J. Michl, ibid., 100, 6861-67 (1978). spectrum. From the observed positive peaks of the $\mathrm{L}_{b}$ band in the MCD spectra we can conclude that here the $\mu^{+}$contributions dominate and that the splitting of the highest two occupied molecular orbitals, $\triangle$ HOMO, is smaller than that of the lowest two unoccupied molecular orbitals, $\triangle$ LUMO. This will result in a $\mu^{+}$contribution to the $\mathrm{L}_{\mathrm{a}}$ band which would reinforce the $\mu^{-}$ contribution and accounts for the strongly negative MCD peak for this transition. It has already been pointed out ${ }^{12}$ that CNDO calculations produce $\triangle \mathrm{HOMO}<\Delta \mathrm{LUMO}$ for the bridged annulenes 5-8, in agreement with this conclusion. Our CNDO calculations now also yield $\triangle H O M O-\triangle L U M O$ values of -0.7 , -0.6 , and $-1.4 \mathrm{eV}$, for 1,2 , and 3 , respectively.

The two contributions to the MCD peak of the $\mathrm{L}_{6}$ transition oppose each other and nearly cancel, accounting for its very low observed intensity. Since the two contributions originate in magnetic mixing of $\mathrm{L}_{b}$ with different excited states, the bisignate shape in the spectra of 1-3 is understandable when one remembers that the effect of the mixing is indirectly proportional to state separation on the energy scale. The $\mu^{-}$contribution is primarily due to the mixing of the $L_{b}$ state with the relatively remote $B_{a}$ state, so that it is roughly constant for all observed vibrational levels of the $\mathrm{L}_{b}$ transition. On the other hand, the $\mu^{+}$contribution is primarily due to the mixing of the $\mathrm{L}_{b}$ state with the nearby $\mathrm{L}_{a}$ state and is considerably weaker in the region of the band origin than in the region of the higher vibrational levels. Thus, in the region of the origin, the MCD sign is imposed by the $\mu^{-}$contribution and at higher energies by the $\mu^{+}$contribution. The resolution is insufficient to determine whether the short-axis polarized vibronic components of the $\mathrm{L}_{b}$ band which borrowed their intensity from the $\mathrm{L}_{\mathrm{a}}$ transition, and which can obtain MCD intensity by magnetic mixing with the $B_{b}$ state, actually contribute significantly to the complex shape of the MCD curve in the region of the $L_{b}$ transition.

While the MCD signs and relative intensities can be understood in the qualitative fashion just outlined, we do not attempt a quantitative analysis. In view of the delicate balancing of effects undoubtedly present, the CNDO-CI model seems to us inadequate for the job. The present qualitative analysis which accounts for all four signs is considerably more satisfactory than the previous simple argument based on the signs of the B transitions alone, in which all next-nearest-neighbor interactions in the $\pi$ system were neglected and in which $\mu^{-}$contributions were absent as a result. The present conclusion is $\triangle H O M O<\triangle L U M O$, and this can be accounted for by consideration of the electron-donating effects of the bridge alone, along the lines discussed in ref 9 , without invoking hyperconjugation, but, of course, not excluding it. A better case for hyperconjugation with the bridge is found in the bridged annulenes 9 and $10 .^{13}$

\section{Conclusions}

The following answers can be provided for the three questions raised in the introduction. (i) Transannular interaction is definitely present in the bridged annulenes 1-3. They can be properly referred to as homonaphthalene, bishomoanthracene, and trishomotetracene, respectively, and viewed as examples of "arrested transition states" along electrocyclic reaction paths. (ii) The spectra of the annulenes $\mathbf{1 - 3}$ are most readily understood by postulating the presence of at least one low-energy transition other than the $\mathrm{L}$ and $\mathrm{B}$ bands. In our opinion, a satisfactory explanation of the origin of the apparent excess of low-energy transitions in large bridged annulenes has yet to be found. (iii) The MCD spectra of 1-3 are in excellent agreement with the general model ${ }^{31}$ for $(4 N+2)$-electron [ $n$ ] annulenes, and the absolute signs agree with the relative magnitudes of $\triangle H O M O$ and $\triangle$ LUMO obtained from $\mathrm{CNDO} / \mathrm{S}$ orbital energies.

Acknowledgment. This work was supported by a NATO Research Grant. The authors at Utah acknowledge support from the U.S. National Science Foundation (CHE 78-27094) and the U.S. Public Health Service (GM 21153). 